\title{
Criminologie
}

\section{Les femmes détenues d'Unité 9 : entre espace fictionnel et réalité}

\section{The female inmates of Unité 9: comparing fictional space and reality}

\section{Las mujeres detenidas de Unidad 9 : entre espacio ficcional y realidad}

\section{Sophie Cousineau et Sylvie Frigon}

Volume 49, numéro 2, automne 2016

URI : https://id.erudit.org/iderudit/1038427ar

DOI : https://doi.org/10.7202/1038427ar

Aller au sommaire du numéro

Éditeur(s)

Les Presses de l’Université de Montréal

ISSN

0316-0041 (imprimé)

1492-1367 (numérique)

Découvrir la revue

Citer cet article

Cousineau, S. \& Frigon, S. (2016). Les femmes détenues d'Unité 9 : entre espace fictionnel et réalité. Criminologie, 49(2), 323-347.

https://doi.org/10.7202/1038427ar
Résumé de l'article

Dans cet article, nous nous penchons sur Unité 9, une télésérie québécoise portant sur l'univers carcéral au féminin, pour dresser un parallèle entre celle-ci et l'incarcération des femmes de ressort fédéral. Dans un premier temps, nous exposons un bref historique de l'enfermement au féminin et nous soulignons quelques particularités inhérentes à cette population carcérale. Nous présentons ensuite le synopsis de la série et nous brossons un portrait qui souligne la pertinence, les avantages ainsi que les lacunes des analyses télévisuelles. Dans un deuxième temps, nous théorisons l'espace carcéral, les dynamiques qui y prennent place ainsi que la résistance entreprise par les femmes (Bosworth, 1999 ; Butler, 1988, 2006a, 2006b ; DeGraff et Kilty, 2016 ; Frigon, 2000, 2012 ; Joël-Lauf, 2009 ; Lavigne, 1999 ; Smoyer, 2016). Dans un troisième temps, nous dressons des liens entre fiction et réalité en explorant quatre espaces : le secteur des admissions, le secteur de l'isolement, les unités résidentielles et les unités mère-enfant. Nous y relevons diverses nuances de résistance, d'aliénation ou de soumission. Ainsi, cet article met en lumière le rapport entre l'espace carcéral et les femmes incarcérées : non seulement celles de ressort fédéral, mais aussi celles d'Unité 9 . 


\title{
Les femmes détenues d'Unité 9 : entre espace fictionnel et réalité
}

\author{
Sophie Cousineau ${ }^{1}$ \\ Candidate au doctorat \\ Département de criminologie, Université d'Ottawa \\ scous070@uottawa.ca \\ Sylvie Frigon \\ Professeure titulaire \\ Département de criminologie, Université d'Ottawa \\ Titulaire de la Chaire facultaire en sciences sociales, Université d'Ottawa \\ Associée de recherche principale \\ Peterhouse, University of Cambridge, Royaume-Uni \\ sylvie.frigon@uottawa.ca
}

RÉSUMÉ - Dans cet article, nous nous penchons sur Unité 9, une télésérie québécoise portant sur l'univers carcéral au féminin, pour dresser un parallèle entre celle-ci et l'incarcération des femmes de ressort fédéral. Dans un premier temps, nous exposons un bref historique de l'enfermement au féminin et nous soulignons quelques particularités inhérentes à cette population carcérale. Nous présentons ensuite le synopsis de la série et nous brossons un portrait qui souligne la pertinence, les avantages ainsi que les lacunes des analyses télévisuelles. Dans un deuxième temps, nous théorisons l'espace carcéral, les dynamiques qui y prennent place ainsi que la résistance entreprise par les femmes (Bosworth, 1999; Butler, 1988, 2006a, 2006b; DeGraff et Kilty, 2016; Frigon, 2000, 2012; Joël-Lauf, 2009; Lavigne, 1999; Smoyer, 2016). Dans un troisième temps, nous dressons des liens entre fiction et réalité en explorant quatre espaces: le secteur des admissions, le secteur de l'isolement, les unités résidentielles et les unités mère-enfant. Nous y relevons diverses nuances de résistance, d'aliénation ou de soumission. Ainsi, cet article met en lumière le rapport entre l'espace carcéral et les femmes incarcérées: non seulement celles de ressort fédéral, mais aussi celles d'Unité 9.

MOTS CLÉS • Espace, Unité 9, levier sociologique, résistance, femmes incarcérées.

1. Département de criminologie, Université d'Ottawa, Pavillon des sciences sociales, 120, rue Université, Ottawa (Ontario), Canada, K1N 6N5.

Criminologie, vol. 49, no 2 (2016) 


\section{Introduction}

Signée par Danielle Trottier, Unité 9 est une télésérie québécoise qui dépeint le quotidien de femmes incarcérées à l'établissement fédéral ${ }^{2}$ de Lietteville. Diffusée sur les ondes de Radio-Canada depuis 2012, Unité 9 a connu un véritable engouement avec un public moyen de deux millions de téléspectateurs pendant la deuxième saison (Numéris, 2015). Le phénomène des représentations télévisuelles de femmes incarcérées n'est pas récent. Il a fait son apparition dans plusieurs pays et à diverses époques, notamment avec Prisoner: Cell Block $H$ (1979-1986) et Wentworth (2013-) en Australie, Bad Girls (1999-2006) en Angleterre, Women in Prison (1987-1988) ainsi qu'Orange is the New Black (2013-) aux États-Unis.

En plus de joindre et de divertir un vaste public, Unité 9 permet de «mieux comprendre la réalité carcérale et les différentes couches de souffrance de ces femmes artichauts» (Frigon, 2013, paragr. 2). Les scènes d'Unité 9 peuvent illustrer des éléments de la situation des femmes incarcérées soulignés par des chercheurs d'ici et d'ailleurs, notamment Hamelin (1989), Frigon (2002, 2004), Frigon et Kerisit (2000), Shantz et Frigon (2010), Giroux et Frigon (2011), le Bureau de l'enquêteur correctionnel (2014-2015), Bosworth (1999), Pollock (2002), Enos (2001), Smoyer (2016), Rostaing (1997), ainsi que Joël-Lauf (2009, 2013). Le recours à cette télésérie met notamment en relief les effets de certaines procédures institutionnelles, telles les fouilles de «bienvenue» chez les femmes incarcérées (Frigon, 2012; Hamelin, 1989). Au-delà de la simple illustration, Unité 9 est mobilisée dans cet article pour explorer le rapport complexe entre l'espace carcéral et les femmes détenues.

Pour ce faire, nous découpons cet article en trois axes. L'axe 1 est de nature contextualisante. Nous présentons le portrait de l'incarcération des femmes de ressort fédéral et le synopsis de la télésérie Unité 9. Nous discutons de la pertinence, des avantages et des inconvénients des analyses télévisuelles. L'axe 2 est de nature théorique: nous conceptualisons d'abord les constituants de l'espace carcéral (Millhaud, 2015; Moran, Pallot et Piacentini, 2013 ; Mund et Bruckert, 2013), les dynamiques qui y sont inhérentes (Chauvenet, 2000; Clemmer, 1958; Goffman, 1961 ; Le Caisne, 2000; Sykes, 1958), ainsi que les stratégies

2. Il y a une distinction à faire entre les établissements provinciaux (sentences inférieures à deux ans) et les établissements fédéraux (sentences supérieures à deux ans). 
empruntées par les femmes incarcérées réunies sous le vocable de résistance (Bosworth, 1999; Butler, 1988, 2006ab; DeGraff et Kilty, 2016; Frigon, 2000, 2012; Joël-Lauf, 2009; Lavigne, 1999; Smoyer, 2016). L'axe 3 est de nature illustrative: nous établissons certains dialogues entre la fiction (Unité 9) et la réalité (parcours des femmes incarcérées au Canada) en fonction de l'espace carcéral. Nous explorons quatre points névralgiques de cet environnement: la salle de fouille ou l'aire d'admission, le secteur d'isolement, l'unité résidentielle et l'unité mèreenfant. Nous y repérons diverses formes d'assujettissement, d'aliénation et de résistance. Le tout permet une meilleure compréhension du rapport complexe entre l'espace carcéral et certaines pratiques ou effets, tant du côté de la réalité que de la fiction.

\section{Axe 1.1 La réalité: les femmes purgeant une peine fédérale au Canada}

Au Canada, à partir de 1934, les femmes recevant des sentences d'incarcération supérieures à deux ans étaient hébergées à l'établissement de Kingston ${ }^{3}$. Elles y étaient séparées de leurs homologues masculins. La centralisation de l'enfermement créait des difficultés, dont l'effritement des liens entre parents et enfants, la non-disponibilité de programmes et services ainsi qu'une classification à sécurité maximale. De 1977 à 1991, une douzaine de suicides y étaient enregistrés (Frigon, 2002). En 1990, La création de choix, un groupe d'étude sur les femmes purgeant une peine fédérale a vu le jour. Son rapport a contribué à la fermeture du pénitencier (le 6 juillet 2000) et à la construction de cinq centres régionaux situés respectivement à Joliette (Québec), Kitchener (Ontario), Abbotsford (Colombie-Britannique), Edmonton (Alberta), Truro (Nouvelle-Écosse), en plus d'un pavillon de ressourcement pour femmes autochtones à Maple Creek (Saskatchewan). En 1994, une émeute à la prison pour femmes s'est soldée par une commission d'enquête présidée par la juge Arbour. Son rapport intitulé La Commission d'enquête sur certains événements survenus à la Prison pour femmes de Kingston (1996) a émis 14 recommandations générales et a tenu des conclusions similaires à celles du groupe La création de choix.

3. Il y avait par ailleurs des ententes fédérales-provinciales permettant ainsi à des femmes de purger leur sentence fédérale dans leur province comme ce fut le cas à Tanguay. 
Ces deux rapports clés en matière d'incarcération au féminin soulignent les besoins particuliers de ce groupe et s'inscrivent dans un vent de réformes. Selon Frigon (2002), ils réitèrent le lien entre victimisation (traumatismes et abus) et criminalisation. Le rapport La création de choix estime que $80 \%$ des femmes ont été victimes de violence. Par ailleurs, le rapport Arbour (1996) mentionne que les deux tiers des femmes de ressort fédéral ont des enfants et que $70 \%$ d'entre elles sont monoparentales. Pour favoriser le maintien des liens entre les mères et leurs enfants, les établissements régionaux sont construits à environ une heure des grands centres urbains et comprennent des programmes mère-enfant.

\section{Axe 1.2 La fiction: Unité 9}

Pour les lecteurs qui ne sont pas familiers avec la télésérie, voici le synopsis des quatre saisons ${ }^{4}$. La première saison gravite autour du parcours carcéral de Marie Lamontagne ${ }^{5}$, une enseignante du secondaire condamnée à purger une sentence au pénitencier de Lietteville pour tentative de meurtre à l'endroit de son père. Elle est placée à l'unité résidentielle 9 qu'elle partage avec d'autres femmes. Alors que Marie s'adapte à son environnement hostile et devient présidente du comité des détenues, ses codétenues rencontrent des obstacles, allant d'une grossesse imprévue, à une dépendance sexuelle assortie à des dettes de drogues, en passant par une tentative de suicide. En revanche, la pionnière de l'unité bénéficie de permissions de sortie avec accompagnatrice. La saison se conclut par une série de rebondissements: un incendie au secteur maximum et l'agression d'une IPL (intervenante de première ligne). Ces deux événements se soldent par une mini-émeute. L'une des résidentes de l'unité 9 profite de cette diversion pour s'évader. Quant à Marie, troublée par le décès de son père, elle s'en prend violemment à une détenue de l'aile psychiatrique. Marie plonge.

À la deuxième saison, Marie se trouve dans un état dépressif. Elle tente de s'en sortir grâce aux bons soins de l'aumônier. Les résidentes de l'unité 9 cheminent: l'une amorce des démarches de libération

4. La cinquième saison est diffusée sur les ondes de Radio-Canada depuis le 13 septembre 2016.

5. Les rôles des personnages principaux sont campés par de grandes comédiennes aimées du public. Par exemple, Guylaine Tremblay incarne le personnage principal de Marie Lamontagne. 
conditionnelle, une autre accouche et est libérée. Nous faisons aussi la connaissance d'une détenue ayant subi une double mastectomie à la suite d'un cancer et d'une femme itinérante qui sème la bisbille au sein de l'unité 9. Plus encore, une rivalité à propos du monopole des drogues à Lietteville se développe entre les unités 7 et 9. Des menaces sont ainsi proférées à l'endroit de clientes endettées. De plus, des stratagèmes sont élaborés pour prendre les rênes du trafic. La saison se termine par une tentative de meurtre à l'endroit de Marie.

À la troisième saison, Marie se rétablit lentement de son incident. Un ancien collègue la demande en mariage et elle accepte. En revanche, l'histoire d'amour entre une détenue du secteur maximal et une IPL et celle entre deux résidentes de l'unité 9 se terminent en queue de poisson. Par ailleurs, après un vol de banque avorté, deux ex-résidentes de l'unité 9 sont réincarcérées à Lietteville. Un diagnostic de cancer est annoncé à la détenue pionnière, mais elle refuse de se faire traiter. Aussi, une résidente de l'unité 9 éprouve une affection non réciproque pour une femme. Rejetée, elle l'agresse verbalement, ce qui pousse cette dernière à commettre une tentative de suicide. Sur une note plus joyeuse, on autorise une intervention chirurgicale de reconstruction mammaire à une détenue de l'unité 9 ; cette dernière s'évade au cours de son hospitalisation. Quant à Marie, elle se rend au domicile de son conjoint pour l'accuser d'agression sexuelle sur sa fille. Parallèlement à ces événements, les deux enfants de Marie succombent dans un accident de voiture.

À la quatrième saison, Marie est anéantie par la nouvelle du décès de ses enfants. Elle porte formellement des accusations en matière d'agression sexuelle contre son conjoint. Elle tente de masquer sa peine en consommant des cachets et en mettant sur pied un trafic de narcotiques. Deux nouvelles IPL sont accueillies, soit une mère et sa fille, de même qu'un AC-3 (agent correctionnel, niveau 3). En raison de problèmes personnels, la chef de la sécurité devient intervenante à l'unité de vie structurée (UVS) où sont hébergées deux anciennes de l'unité 9. À l'hôpital, une détenue se remet lentement d'une récente agression à la pelle. Elle rejoint bientôt l'UVS. Par ailleurs, une femme de l'unité 9 accouche de jumeaux et bénéficie d'une libération conditionnelle. Le bras droit du caïd de Lietteville, une détenue frustrée par l'inertie de l'IPL responsable de son plan correctionnel, assassine brutalement cette dernière. Cet événement bouleverse l'établissement. L'unité 9 accueille quatre nouvelles détenues: une femme hispanique, une avocate crimi- 
naliste, une religieuse et une jeune adulte, ce qui donne lieu à de nouvelles dynamiques. De son côté, Marie se voit accorder un séjour au secteur des visites familiales privées (VFP) où elle orchestre une tentative de suicide. Parallèlement, le caïd de l'unité 7 s'en prend violemment au directeur.

\section{Axe 1.3 Entre les deux: un dialogue}

Plusieurs scientifiques sociaux mobilisent des représentations artistiques, notamment des téléséries, en tant que matériaux de recherche. Winckler (2009) prête de nombreux avantages à celles-ci comparativement aux émissions d'actualité. Selon lui, elles constituent de puissants mécanismes pour diffuser des idées. À l'exception des téléséries en France qui sont censurées, celles produites ailleurs «interpellent constamment la situation sociale, politique, idéologique du monde où vit son public» (paragr. 9). En plus d'être engagés et d'ouvrir un dialogue durable avec les sujets d'actualité, les personnages mis en scène dans les téléséries peuvent représenter une diversité culturelle de même qu'une myriade de points de vue sur des sujets éthiques.

Le recours à la télésérie The Wire (2002-2008) a marqué un tournant dans cette tradition. Selon Holt et Zundel (2014), cette télésérie est pertinente parce qu'elle dégage une certaine passion (capacité de conviction), elle évoque le monde de l'intérieur (pouvoir d'expression) et elle montre l'aspect humain des choses à travers un fil événementiel (tragédie). Ces trois mécanismes de représentation permettent de communiquer à un auditoire des éléments à portée sociologique. De plus, cette transposition prend la forme d'un lyrisme sociologique. Ainsi, les sentiments associés à une situation peuvent pénétrer le cœur et l'imaginaire d'un public (Penfold-Mounce, Beer et Burrows, 2011).

Comme le synthétise Frigon (2013), «ces femmes pourraient être nos sœurs, nos mères, nos filles, nos amies, même nos grands-mères. Donc cette identification peut être très efficace et servir de levier pour provoquer un élan d'empathie» (paragr. 9). Conséquemment, ces effets de sensibilisation et d'humanisation peuvent servir de tremplin à une transformation sociale. Ce potentiel est d'autant plus grand que les téléséries peuvent interpeller un public cible hors du milieu universitaire (Penfold-Mounce, Beer et Burrows, 2011). Ainsi, Unité 9 peut être un prélude à des pétitions et à des commissions d'enquête qui appelleront à réviser les procédures et les conditions carcérales pour mieux répondre 
aux besoins des femmes. Les usages des téléséries et, de manière plus générale, des représentations artistiques, sont multiples. Elles peuvent s'avérer de puissants outils pédagogiques pour sensibiliser, éduquer ou autonomiser les gens (Knowles et Coles, 2008), ou elles peuvent aussi servir de porte-voix aux groupes marginalisés (Leavy, 2009).

Malgré cette portée sociale et ses utilisations plurielles, le recours à des téléséries comme Unité 9 n'est pas dépourvu d'angles morts. Comme l'objectif derrière une télésérie de ce genre est de captiver et de conserver un public d'envergure au fil de ses saisons, elle peut avoir des tendances sensationnalistes, et dans le cas d'Unité 9 , à surreprésenter les occurrences de violence et d'abus en ce qui a trait à leur forme et à leur contenu. Par exemple, pas moins de quatre membres du personnel ont des rapprochements intimes avec les détenues. Plus encore, les mécanismes de plaintes et de griefs sont passés sous silence. Il en est de même pour le Protecteur du citoyen. Somme toute, la télésérie peut nourrir des préjugés chez le public à propos de la prison.

En fait, puisque certaines téléfictions sont subventionnées par l'État, elles peuvent diffuser des messages payants pour les autorités ou renforçant le statu quo. Puisque Lietteville est dépeint comme un établissement qui connaît des sévices, des règlements de compte et l'enfer des drogues, Unité 9 s'avère un puissant mécanisme de dissuasion générale. Pourtant, ce type de discours est partiel et partial. D'autres discours sont tout aussi légitimes et admis: certaines femmes incarcérées accordent une connotation positive à la prison en la percevant comme un lieu familier, un moment de répit ou l'occasion de renouer avec leur réseau social.

Enfin, comme il est question de représentations, le contenu n'est pas composé intégralement d'expériences ou de propos rapportés. Nous reconnaissons toutefois qu'en ce qui concerne Unité 9, l'auteure Danielle Trottier a tout de même fait de la recherche sur le terrain pendant plusieurs années avant de se lancer dans la rédaction de la série.

\section{Axe 2.1: Les particularités de l'espace carcéral}

Les espaces se déclinent de différentes manières dans l'environnement carcéral. Selon Milhaud (2015), l'espace carcéral est à la fois un agent actif de l'enfermement, de l'organisation des pratiques (gestion du risque) et de la division des activités. Ces endroits témoignent de la conciliation de l'exclusion et de la réinsertion. Les établissements car- 
céraux sont aménagés de sorte à favoriser la circulation, la répartition et la visibilité des individus à des fins de sécurité et de surveillance. L'architecture carcérale est l'un des piliers du dispositif de surveillance théorisé par Foucault: le Panopticon (Milhaud, 2015).

Selon nous, certains espaces de la prison participent à la mortification du sujet. On fait prendre conscience au reclus de son statut d'inférieur et de soumis (Goffman, 1961). C'est ce que représente le secteur d'admission avec ses procédures de dépouillement et de mise à nu. Quant au secteur d'isolement, il s'agit d'un espace multifonctionnel, servant notamment à briser la résistance des détenus.

En ce qui concerne sa caractérisation, il s'agit surtout d'un espace pénal public. Les frontières traditionnelles entre public et privé sont brouillées. Des parcelles d'intimité sont réappropriées par certains détenus à même l'espace public. De telles stratégies témoignent de leur agentivité (Moran, Pallot et Piacentini, 2013). Plus encore, les cellules sont perçues comme un espace sécuritaire et familier aux yeux des détenus (Baer, 2005; Mund et Bruckert, 2013). Quant aux secteurs ou établissements à sécurité maximale, moyenne ou minimale, ils possèdent des infrastructures qui permettent des déplacements ou des contacts en fonction du degré de dangerosité du détenu.

En plus de prescrire des usages, nous croyons que l'espace produit des effets chez les reclus, dont une docilisation et une régulation des corps (Millhaud, 2015; Moran, Pallot et Piacentini, 2013). Bien sûr, le détenu qui circule dans l'espace carcéral doit aussi interagir avec autrui. Ce faisant, il répond à une logique et à des prémisses façonnées par cet environnement. Puisque la prison produit une coupure entre le sujet et le monde extérieur, une société de rechange s'y développe. Le détenu ainsi privé de liberté doit composer avec des pertes en termes de biens et de services, d'autonomie, de sécurité, de relations hétérosexuelles, etc. Pour pallier ces pertes, les détenus adoptent des rôles sociaux (Sykes, 1958). Cette classification sociale prend aussi la forme d'une hiérarchisation des rapports ou la formation de classes en fonction du crime commis, de la propension à dénoncer ou de sa position par rapport au système de privilèges (Chantraine, 2004; Clemmer, 1958; Le Caisne, 2000). Une réappropriation de l'espace est conséquente à cette hiérarchisation: certaines tâches sont réservées aux individus au bas de la hiérarchie sociale tandis que ceux au sommet en sont exemptés. De plus, les rats (délateurs) se retrouvent fréquemment au secteur d'isolement cellulaire (à la protect). Ce même système de privilèges 
organise aussi les rapports avec l'administration et le personnel (Chauvenet, 2000; Sykes, 1958). Il fonctionne selon une logique donnant-donnant et il traduit une capitalisation des avoirs tant par les détenus que par l'administration. Il permet aux deux parties de mobiliser une parcelle de pouvoir et d'organiser les rapports de force qui en découlent.

Par conséquent, ces mêmes endroits incitent parfois à la résistance ou à l'agentivité chez les femmes incarcérées. Smoyer (2016) affirme que la résistance est une dimension centrale de l'incarcération et un élément clé à sa compréhension. La résistance y est négociée, multiforme (insidieuse ou visible, désorganisée ou non) et s'exerce par rapport au pouvoir institutionnel (ses représentations ou ses règlements). La résistance entreprise par les femmes incarcérées vise un gain de contrôle sur leur existence. Pour Frigon (2000), le «corps-résistance» en prison caractérise diverses stratégies «pour se réapproprier un sens d'identité ${ }^{6}$ (p. 145). Celles-ci comprennent entre autres l'art de la survie et l'automutilation. Grâce au premier, les femmes font le lien entre leur oppression et leur corps. Par la seconde, les femmes acquièrent un certain contrôle sur leur corps et son marquage. Lavigne (1999) précise que les comportements de résistance sont souvent perçus par l'institution comme étant perturbateurs. Ils caractérisent aussi d'autres tactiques tels le sommeil, la formation de solidarités et la transformation de leur cellule en espace pour soi ${ }^{7}$. À la fois De Graaf et Kilty (2016), Smoyer (2016) et Joël-Lauf (2009) font référence au concept de résistance pour pallier les privations de l'environnement carcéral ou les contraintes institutionnelles. Joël-Lauf (2009) examine comment les détenues transforment les parloirs en espace fécond à leur sexualité. Lors des repas, les femmes incarcérées pallient les privations en matière de biens et services en consignant, en partageant et en échangeant des denrées alimentaires (De Graaf et Kilty, 2016) ${ }^{8}$. De telles activités leur permettent

6. Cette même idée se retrouve chez Bosworth (1999). La résistance se traduit par le discours des détenues qui se redéfinissent en tant que mères par l'entremise de pratiques non normatives ou qui réclament leurs besoins particuliers de femmes aux surveillants à même l'espace public.

7. Baer (2005) observe que les détenus décorent leurs cellules à l'aide de lotions et de babioles pour les personnaliser, témoigner de leur pouvoir d'achat à la cantine et exposer le legs d'anciens détenus.

8. Aux États-Unis, Smoyer (2016) explore le rapport entre les denrées alimentaires et les formes de résistance entreprises par les femmes incarcérées. 
de choisir le moment où elles s'alimenteront et de redéfinir la nourriture en tant que monnaie d'échange dans le système carcéral.

Enfin, Butler (1988) caractérise plus largement la résistance, celle-ci étant surtout performative. La redéfinition ou la subversion se traduit aussi par des pratiques à l'extérieur de la matrice hétérosexiste, une structure de pouvoir en double inversé traversée par des liens causaux entre les catégories genre, sexe, désir et orientation sexuelle. Ce faisant, la résistance caractérise les sexualités périphériques et tout ce qui va à l'encontre des normes traditionnellement associées à la masculinité ou à la féminité.

\section{Axe 3.1 La salle de fouille et le secteur des admissions}

Disons tout d'abord que ces espaces sont marqués par un «rite d'entrée» (Frigon, 1999). Il s'agit de la fouille de bienvenue, vocable repris d'ailleurs par certains des personnages d'Unité 9, à la fois détenues et IPL. Cette fouille fait partie d'un processus que Goffman (1961) nomme la cérémonie d'admission. Par une série de procédures, dont l'enregistrement et la consignation des biens, la prise des empreintes digitales et de photos ainsi que l'attribution d'un numéro de matricule, la détenue prend conscience de son appartenance à l'institution. En bref, elle devient une détenue. Cet effet hautement symbolique est amplifié par la remise d'un uniforme. Les pénitenciers canadiens échappent à cette règle. Cependant, si la détenue ne possède pas de vêtements de rechange au moment de son arrivée, comme c'est le cas de Marie dans Unité 9 , elle reçoit une trousse de dépannage constituée de vêtements génériques (Saison 1, épisode 2).

Par ailleurs, la cérémonie d'entrée comprend une fouille à nu. Ceci est motivé par un énoncé de mission qui va comme suit:

Le Service correctionnel du Canada, en tant que composante du système de justice pénale et dans la reconnaissance de la primauté du droit, contribue à la sécurité publique en incitant activement et en aidant les délinquants à devenir des citoyens respectueux des lois, tout en exerçant sur eux un contrôle raisonnable, sûr, sécuritaire et humain. (Service correctionnel Canada, 2013, sect. 1)

Dans un tel cadre, les pratiques comme la fouille sont justifiées. La présomption d'innocence est ici renversée: jusqu'à preuve du contraire, le corps camoufle potentiellement de la contrebande ou des drogues, et 
constitue un risque à la sécurité de l'établissement. Ainsi, ces fouilles permettent de détecter la présence de matériel.

Néanmoins, ce procédé a des effets. Il est vécu en tant que violence sexuelle par celles qui le subissent (Hamelin, 1989). Le viol et la fouille à nu sont deux expériences qui partagent plus d'un lieu commun: pouvoir vertical (coercition), aspect unilatéral, absence de libre arbitre. À l'instar de Garfinkel (1976), Frigon (2012) est d'avis qu'en raison de ces aspects, la fouille à nu est une «cérémonie de dégradation» qui marque le corps (p. 231). Bien sûr, le rapport Arbour (1996) a recommandé que la fouille à nu soit pratiquée uniquement par des agentes correctionnelles. Néanmoins, ses effets traumatisants persistent. Dans Écorchées, les effets de la fouille sont illustrés ainsi: «[elle] se rappelle une fouille vaginale-rectale subie il y a quelques années. "Ouvre la bouche, lève les bras, lève les pieds, ouvre les jambes, écarte les fesses.» Humiliation. Contrôle» (Frigon, 2009, p. 74).

À l'épisode 2 de la saison 1, Marie doit se soumettre elle aussi à une fouille de bienvenue. Son regard se voile de larmes et sa bouche se crispe lorsqu'elle doit obéir aux ordres d'une IPL. Son corps est scruté par les regards de deux membres du personnel. À la saison 4, épisode 13 , survient une scène particulièrement saisissante: une ex-religieuse, âgée et de surcroît extrêmement pudique, vit difficilement la fouille de bienvenue, et ce, malgré la main réconfortante d'une agente posée sur son épaule. On lui retire ses appareils auditifs et son dentier afin de les examiner. Ce dépouillement renforce l'effet dégradant de la procédure. S'il y a soumission chez les détenues précédentes, une détenue anciennement itinérante refuse quant à elle d'obtempérer aux ordres. Son refus de se dévêtir entraîne l'intervention musclée de quatre IPL. Sa résistance est de courte durée. Elle se soumet rapidement aux représentants de l'institution.

Somme toute, vécu souvent comme un «viol» de l'intimité, cet assaut intervient dans un processus plus large de mortification du soi qui vise à transformer le citoyen en sujet institutionnel. Plus encore, cette procédure peut s'avérer un test pour vérifier le degré de soumission de la détenue et lui rappeler qui est le détenteur du pouvoir (Goffman, 1961). C'est le message envoyé aux détenues d'Unité 9 qui refusent d'obéir aux ordres des IPL lors de la fouille. En raison de ses pratiques et de ses symboles, cet espace accentue la servilité des détenues et laisse peu de place à la résistance. 


\section{Axe 3.2 L'isolement cellulaire}

En milieu carcéral, l'isolement cellulaire prend généralement deux formes: l'isolement disciplinaire et l'isolement préventif (administratif) (Chamberland, 2014; rapport Arbour, 1996; Société Elizabeth Fry du Québec, 2005). L'isolement disciplinaire répond à une entorse aux règlements de l'établissement. En ce qui concerne les prisons québécoises, son application s'inscrit dans un processus disciplinaire constitué selon Chamberland (2014) de sept étapes, allant du signalement d'un manquement par un agent correctionnel à sa revue par un comité disciplinaire, et se soldant par l'imposition d'une sanction au détenu, dont le confinement ou la réclusion. «Le confinement est l'isolement de l'individu dans sa propre cellule, sur son secteur habituel, pour une période maximale de 5 jours, alors que la réclusion est l'isolement de l'individu dans une cellule spécifiquement prévue à cet effet, située dans une aile particulière du centre de détention» (Chamberland, 2014, p. 22). À la suite d'un manquement, il est aussi possible de placer le détenu en isolement cellulaire jusqu'à concurrence de 24 heures.

En revanche, l'isolement préventif ou administratif peut être invoqué pour plusieurs motifs visant à maintenir la sécurité de l'établissement. Les détenues peuvent aussi en faire la demande si elles craignent une atteinte à leur propre sécurité. Il peut servir de dispositif thérapeutique pour prévenir le suicide et les comportements d'autoblessure. Il peut aussi servir de cellule sèche le temps que la détenue évacue naturellement le matériel de contrebande. Les prisons québécoises révisent cette mesure indéterminée de manière hebdomadaire et suivent un protocole (Chamberland, 2014).

D'une part, l'isolement cellulaire est souvent perçu comme une violation de l'article 12 de la Charte canadienne des droits et libertés: le traitement ou la peine cruelle et inusitée. Ce placement provoque souvent une détresse psychologique. Plusieurs auteurs remarquent qu'une telle intervention augmente le risque suicidaire ${ }^{9}$ et les comportements d'autoblessure ${ }^{10}$. Selon le Bureau de l'enquêteur correctionnel,

9. Suto et Arnaut (2010) effectuent un constat similaire. Neuf des vingt-quatre participants interviewés ont commis une tentative de suicide lors de leur placement en isolement cellulaire.

10. Comme nous l'avons mentionné plus tôt, Frigon $(2010,2012)$ voit l'automutilation comme une forme de résistance. Contrairement à leurs expériences antérieures de victimisation, ces pratiques permettent aux détenues d'en contrôler les aspects qualitatifs et quantitatifs. 
«il est inquiétant de constater qu'un certain nombre des suicides en établissement continuent d'être commis dans les cellules d'isolement ou d'observation [...]. La plupart des personnes qui s'enlèvent la vie en prison ont des antécédents de problèmes de santé mentale, de tentatives de suicide ou d'automutilation» (Bureau de l'enquêteur correctionnel [BEC], 2013-2014, paragr. 9). Ceci dresse un parallèle avec le décès en établissement d'Ashley Smith, pour qui le recours à l'isolement préventif visait à gérer les comportements perturbateurs d'autoblessure. «[C]es mesures [...] étaient parfois beaucoup trop restrictives. [...] Elle passait de longues heures dans sa cellule sans aucune stimulation, pas même un livre ou un bout de papier pour écrire» (BEC, 2008, section 2.1.2, paragr. 38). Kilty, LeBlanc et Frigon (2015) font des observations similaires concernant le traitement d'Ashley Smith. Aux yeux de Chartrand (2015), le placement en isolement cellulaire prend la forme d'une violence institutionnelle.

En ce qui concerne les effets victimisants associés à l'isolement, à la saison 4, la détenue soupçonnée d'homicide à l'endroit d'une IPL est placée dans ce secteur le temps qu'une enquête de la Sûreté fasse la lumière sur les faits. Nous sommes témoins de sa décompensation: ses crises de larmes, ses plaintes et son incapacité à gérer ces privations. De plus, l'isolement s'avère un moyen utilisé par le directeur pour «casser» la forte personnalité de l'avocate criminaliste admise à la saison 4. Marie y passe aussi de courts séjours, notamment lorsqu'elle est accusée à tort de possession de matériel de contrebande ou qu'elle présente un risque suicidaire. À la saison 3, ce séjour amplifie ses symptômes de dépression.

D'autre part, une minorité de femmes incarcérées voient l'isolement cellulaire sous l'angle de la résistance (Frigon, 2012; Martel 2001). Pour ces dernières, il constitue un choix éclairé. Puisque ces séjours sont délibérés, ils peuvent s'avérer moins difficiles pour ces dernières. L'isolement peut répondre à un besoin de retrait ou de solitude. Dans Unité 9 , une femme du pavillon résidentiel demande à être placée en isolement préventif pour mettre un terme au harcèlement d'une IPL. La cellule d'isolement devient un refuge.

D'autres comportements exhibés dans cette cellule tiennent de la résistance, ce qui est considéré comme perturbateur par l'administration (Lavigne, 1999). À la suite d'une altercation avec la bibliothécaire, une détenue est placée en cellule d'isolement. Elle entame avec succès une grève de la faim afin que Steven, le psychiatre de l'établissement, lui 
fasse la lecture des pages manquantes de son roman. À la saison 2, la grève de la faim de Marie lui permet de transgresser certains règlements administratifs: l'aumônier lui apporte de la nourriture de l'extérieur.

Par ailleurs, la résistance prend la forme d'activités physiques afin de gérer l'effet claustrophobique d'un tel environnement. C'est ce que fait le caïd de l'unité 7 en improvisant une séance de kickboxing. Bien que cette détenue n'ait aucun contrôle sur son placement en isolement, elle peut néanmoins gérer ses mouvements, sa posture et son rythme cardiaque. Ceci vient à l'encontre de l'aspect disciplinaire de cet espace. Enfin, à la saison 3, lorsque sa chambre est fouillée, une détenue avale une note d'amour dévoilant son idylle avec une IPL. La chef de la sécurité suspecte plutôt qu'elle a ingéré des drogues et la transfère en cellule sèche. Son corps résiste: elle souffre de constipation. Elle doit faire appel à Steven, le psychiatre, pour y remédier et ainsi mettre fin à son séjour. Dans ces trois scénarios, le corps devient un site de résistance (Frigon, 2012).

Ainsi, dans Unité 9, on retrouve les deux types d'isolement cellulaire. En fonction du contexte, les personnages y font l'expérience d'une violence institutionnelle ou d'une retraite. Ils ont néanmoins la possibilité de recourir à diverses stratégies pour déstabiliser le cadre carcéral et l'aspect punitif inhérent à ce dispositif.

\section{Axe 3.3 Les unités résidentielles}

À l'intérieur des pénitenciers canadiens, les femmes ayant une cote de sécurité moyenne ou minimale sont logées dans des unités résidentielles. En raison de leur architecture notamment, ces jolies maisonnettes semblent participer à un objectif plus large de contrôle de type camouflage à la manière d'une «répression dans un gant de velours» (Frigon, 2004, p. 367). Cela amène un élargissement du filet pénal, facilite une surveillance insidieuse et peut se solder par la mise en place d'une structure spatiale qui renforce l'association entre les femmes et leur domestication: leur responsabilisation par l'accomplissement de tâches ménagères ${ }^{11}$. Ce faisant, les stéréotypes genrés sont imposés aux femmes.

11. Par ailleurs, cette forme de responsabilisation offre de l'autonomie et peut aider à freiner, un tant soit peu, l'effet d'institutionnalisation. 
Au sein même de l'unité, la dynamique est sensiblement la même: division des tâches ménagères ${ }^{12}$, buanderie, préparation des repas grâce à une épicerie hebdomadaire de trente-cinq dollars. Les femmes doivent s'autogérer, voire discipliner celles qui ne contribuent pas au bon fonctionnement de l'unité en échouant à garder les aires communes propres. Au cours de la deuxième saison, les femmes de l'unité résidentielle 9 accueillent une détenue à l'hygiène douteuse qui refuse de collaborer aux activités de la maisonnette. Ce faisant, elle s'expose aux sanctions sociales des autres femmes jusqu'à son expulsion. Les actions collectives entreprises par ces femmes pour arriver à ce dénouement sont d'ailleurs caractérisées par une forme de résistance. Grâce à ces actions, elles regagnent un certain contrôle sur le quotidien à l'unité et connaissent une gestion plus harmonieuse.

En plus des effets de domestication ou de résistance, les unités résidentielles sont caractérisées par l'aliénation du corps des femmes incarcérées. Cela s'explique en partie par les limites qui leur sont imposées en termes de budget alimentaire. Le cas présent, ces détenues ne peuvent avoir accès à certaines denrées ou aliments riches en vitamines et minéraux. Les options végétaliennes ou biologiques sont elles aussi limitées. Une enquête menée en 2006 à l'Établissement de Grand Valley a révélé quelques lacunes en matière de services alimentaires (Service correctionnel du Canada, 2006, sections 7.1 à 7.3). Par ailleurs, de nouvelles mesures du Service correctionnel du Canada, comme la standardisation des aliments (préparés, congelés, expédiés) et la transition vers le lait en poudre, amplifient les privations alimentaires. (Clancy, 2015; Mac Farlane, 2015).

Dans Unité 9, l'absence de diversité alimentaire se fait ressentir. Combien de fois une détenue de l'unité 9 se plaint-elle du manque d'assaisonnement et de son régime composé principalement de féculents, à savoir des pâtes, du pain et du riz? Encore à la saison 4, épisode 6, un personnage déplore cette lacune: «j't'écœurée d'manger du maca, faut s'faire d'autre chose pour souper» (34: 58). Cela se solde par ce que Frigon (2012) nomme une désertification du goût. L'aliénation ne se limite pas au goût: «[t]ous les sens sont perturbés» (p. 235).

12. Bien sûr, cette division est coextensive à un système et à la hiérarchisation de la population carcérale. Les chefs d'unité ou les matrones en sont dispensées. Des tâches modifiées peuvent être attribuées aux détenues ayant des problèmes de santé ou aux aînées. 
En revanche, l'aménagement des unités résidentielles rend possibles les rapprochements intimes, notamment ceux de nature homoérotique ${ }^{13}$. Ces pratiques ont le potentiel de déstabiliser le cadre hétérosexiste de Butler (1988) qui prend la forme d'un double inversé. Cette matrice illustre les catégories genre (féminin, masculin), sexe (homme, femme), orientation sexuelle (hétérosexuelle) et désir (monogame) comme ayant une logique causale. Il est possible d'observer soit une forme d'assujettissement, soit une forme de résistance chez les femmes formant une union homoérotique ${ }^{14}$, et ce, en fonction de la logique mobilisée et des activités reproduites.

Règle générale, selon Ward et Kassebaum (1965) et Forsyth, Evans et Foster (2002), de telles unions traduisent plutôt une compulsion au modèle hétérosexiste. D'abord, Ward et Kassebaum (1965) remarquent que la plupart des couples dans les prisons tendent à mimer une structure complémentaire: l'une adopte une attitude et exécute des tâches traditionnellement masculines tandis que l'autre a des comportements typiquement féminins. Pour leur part, Forsyth et al. (2002) remarquent que les discours reproduisent les stéréotypes genrés et l'hétérosexualité institutionnelle: les motifs économiques qui sous-tendent ces relations mettent en valeur la dépendance économique des femmes. Les participantes ont tendance à conceptualiser l'hétérosexualité comme sexualité légitime et normative tandis que l'homosexualité est vue comme temporaire et instrumentale. De telles perceptions renforcent la structure hétéronormative.

En ce qui concerne Unité 9, cela se solde surtout par des transactions qui renforcent la dépendance et l'exploitation sexuelle de certaines femmes au profit d'autres. Par exemple, une détenue échange des séances sensuelles dans la douche ou des nuits érotiques contre des drogues. Ce troc lui permet de se dissocier psychologiquement des difficultés entourant la garde de ses enfants. Une autre détenue se «paie» des services érotiques en offrant des remèdes pseudo-médicinaux. Elle compense ainsi sa poitrine mutilée en renouant avec celle d'autres femmes le temps d'un échange.

13. Comme le suggère Corriveau (2007): «[ce terme dynamique] renvoie à des comportements entre individus de même sexe plutôt qu'à une façon d'être, voire à un personnage défini uniquement par son orientation sexuelle» (paragr. 1).

14. Il va de soi que l'espace est un facteur clé à l'existence de comportements homoérotiques, ces derniers étant plus florissants dans les endroits dits privés ou semi-privés, tels que les douches ou les cellules/chambres à coucher. Divers codes de conduite et degrés de tolérance sont prescrits en fonction de la qualification de l'espace (Joël-Lauf, 2013). 
Cependant, ces contacts peuvent être interprétés comme une forme de résistance ${ }^{15}$. Il y a bien sûr une prise en charge de certaines pulsions sexuelles par des femmes de l'unité 9 et donc une réappropriation de leur désir. De plus, ces rapprochements peuvent minimiser les privations institutionnelles inhérentes à l'enfermement et à ses effets aliénants. À la saison 3, deux femmes se coiffent à l'occasion du mariage de Marie, la première faisant la mise en plis de la seconde dans sa chambre. Cette caresse fait naître un sentiment-surprise de désir coupable. En contraste, à l'épisode 6 de la première saison d'Orange is the New Black, une femme pleure alors que la coiffeuse lui lave les cheveux. Elle ressent non pas des sentiments romantiques ou érotiques, mais bien un manque en matière de contact humain.

Enfin, les rapprochements homoérotiques correspondent à l'idée du corps comme site de résistance (Frigon, 2012) parce qu'ils traduisent une certaine résilience. Cette intimité s'avère une occasion où les détenues peuvent renouer positivement avec leur corps. Ces rapprochements inscrivent dans la chair et dans la mémoire une expérience positive; un fossé se crée alors entre ces nouvelles expériences et les expériences de violence sexuelle vécues antérieurement.

Somme toute, l'unité résidentielle permet certains échanges intimes entre femmes, ceux-ci étant perçus sous l'angle de la résistance ou de l'assujettissement. Quoi qu'il en soit, cet espace participe à l'aliénation sensorielle des participantes et possède un double effet de domestication/responsabilité en raison des tâches à accomplir.

\section{Axe 3.4 L'unité mère-enfant}

Selon le BEC (2013-2014), «trois détenues sur quatre ont aussi des enfants de moins de 18 ans» (section 5, paragr. 5) ${ }^{16}$. Pour maintenir le lien de ces femmes avec leur parentalité, les établissements fédéraux possédaient, à partir de 2001, des unités mère-enfant ${ }^{17}$. Selon les modalités du programme, les enfants pouvaient visiter leur mère les fins de semaine et les jours fériés jusqu'à l'âge de 12 ans ou être en cohabitation

15. Lorsqu'ils prennent place à même l'espace public, ils marquent une forme de résistance, car ils sont qualifiés de comportements perturbateurs par l'établissement (Lavigne, 1999). L'indécence publique fait partie des huit types de manquement disciplinaire mis en exergue par les prisons québécoises (Ministère de la Sécurité publique, 1999, p. 48).

16. Près du tiers des personnages principaux d'Unité 9 sont mères.

17. Ces quartiers désignés sont à dissocier des salles réservées à des activités familiales pendant les fins de semaine et accessibles aux mères et aux grands-mères. 
permanente avec cette dernière jusqu'à l'âge de 4 ans. Seules les femmes ayant une cote de sécurité minimale et moyenne pouvaient y participer (Service correctionnel Canada, 2007). En 2008, d'importantes modifications ont été apportées: l'âge d'admissibilité à temps partiel de l'enfant est passé de 12 à 6 ans, les femmes ayant commis des crimes graves accompagnés de violence, de nature sexuelle ou à l'endroit d'enfants ne pouvaient plus y participer, et d'autres exigences s'y sont ajoutées (voir Wesley, 2012, p. 22). Cela a eu pour effet de réduire considérablement l'accès au programme mère-enfant, surtout en ce qui concerne les détenues autochtones. En mars 2011, il n'y avait qu'une seule participante à l'Établissement Joliette (voir Wesley, 2012, p. 22) ${ }^{18}$. Dans Unité 9 , une seule mère semble participer à un tel programme. Une détenue accouche d'un enfant à la saison 2, mais elle ne peut le garder avec elle, en raison des accusations d'incendie criminel qui pèsent contre elle.

Ainsi, ces unités sont théoriquement censées pallier les privations et la perte de contact avec leurs enfants. Or, leur sous-utilisation contribue à un problème plus large: l'aliénation des détenues par rapport à leur rôle de mère, surtout en ce qui concerne la grossesse et la maternité (Frigon, 2012). Elles éprouvent aussi certaines difficultés dans la coordination des contacts téléphoniques et des visites avec leurs enfants (BEC, 2013-2014). Cet amenuisement des liens survient donc sur plusieurs fronts.

Somme toute, les unités mère-enfant gagneraient à être mieux gérées. Ces espaces portent en eux un potentiel de résistance et témoignent d'un gain de contrôle par les femmes, de leur autonomisation en tant que mères, de la réappropriation d'un contact régulier avec leur enfant ainsi que d'une capacité à poser des choix quotidiens en matière de soins et d'éducation de leur enfant. Ce faisant, ces éléments font écho à la plupart des principes directeurs ${ }^{19}$ du groupe d'étude La création de choix, qui ont d'ailleurs été repris par Service correctionnel Canada en tant que philosophie organisant l'enfermement des femmes de ressort fédéral.

18. Brennan (2014) présente des statistiques et un constat similaires.

19. Ces cinq principes directeurs sont les suivants: pouvoir contrôler sa vie, des choix valables et responsables, respect et dignité, environnement de soutien, responsabilité partagée (Service correctionnel Canada, 2015). 


\section{Conclusion}

À l'aide de quatre espaces inhérents au milieu carcéral, nous avons dressé un parallèle entre la réalité de l'incarcération des femmes au Canada et celle des personnages d'Unité 9. Le secteur de la fouille et des admissions, le secteur d'isolement, les unités résidentielles et l'unité mère-enfant donnent lieu à divers degrés de soumission, d'aliénation ou de résistance. Ils témoignent d'un rapport complexe entre les femmes et l'espace carcéral.

Bien que la télésérie Unité 9 soit hautement fertile en matière de rapprochements avec la réalité, il existe des écarts. L'image projetée y est partielle car Unité 9 brosse l'univers d'un établissement de ressort fédéral. Ce faisant, elle passe sous silence les établissements provinciaux (deux ans moins un jour) et les pavillons de ressourcement pour personnes autochtones. De plus, les femmes appartenant aux Premières Nations, à des minorités culturelles ou trans ${ }^{\star 20}$, surreprésentées dans notre système carcéral ${ }^{21}$, sont relativement absentes de la télésérie. Par exemple, une seule femme autochtone est entraperçue avant le placement fédéral d'une détenue à la quatrième saison.

En revanche, les minorités culturelles et d'identité de genre sont représentées dans la série étasunienne Orange is the New Black. Les dortoirs sont séparés en fonction de l'appartenance culturelle des femmes. Quant à la détenue Sophia, transsexuelle ${ }^{22}$, elle évoque les enjeux en matière de discrimination et de transition ${ }^{23}$. Si des personnages appartenant à des minorités culturelles sont représentés dans

20. L'astérisque renvoie à la pluralité des identités de genre et d'expression du genre qui peuvent être incorporées sous ce terme. Voir Killermann (2012) pour en savoir davantage sur l'origine et les emplois associés à ce terme.

21. «Bien que les peuples autochtones représentent $4,3 \%$ de la société canadienne, $24,6 \%$ de la population carcérale totale actuelle est composée d'Autochtones, et les femmes autochtones représentent maintenant $35,5 \%$ de la population de femmes détenues. Dans cette même période, le nombre de détenus issus de la population noire a augmenté de $69 \%$. Le taux d'incarcération dans un établissement fédéral de personnes issues de la population noire est trois fois plus élevé que leur taux de représentation dans la société en général» (BEC, 2014-2015, p. 6). Par ailleurs, en raison de leur précarité socioéconomique, les personnes trans ${ }^{\star}$ sont plus à risque d'avoir des démêlés avec le système de justice pénale. Or, le SCC ne compile pas de statistiques officielles sur ce groupe (PrisonJustice.ca, 2016). PASAN, un organisme à but non lucratif, a mené une étude (1993 à 1999) dans laquelle $10 \%$ des détenus de leur échantillon ayant le VIH/SIDA étaient trans ${ }^{\star}$.

22. Bien sûr, un seul cas de figure est couvert: la transition d'homme à femme.

23. Interprétée par Laverne Cox, cette actrice ouvertement trans ${ }^{\star}$ agit dorénavant à titre de porte-parole de la communauté. En 2014, l'organisme à but non lucratif GLAAD lui a remis un prix pour souligner son implication au sein de la communauté trans ${ }^{\star}$. 
Unité 9, les clichés sont repris. Le caïd de Lietteville, une femme d'origine jamaïcaine, de surcroît anglophone, fait preuve d'accès de rage. Le premier personnage hispanique, une femme ultra religieuse, apparaît à la quatrième saison.

Si Unité 9 aborde les questions trans ${ }^{\star}$, elle le fait d'une manière qui pose problème $e^{24}$. Elle les conceptualise comme une erreur (déviance) à corriger et elle les interprète par un discours médicalisant ou psychologisant. Une détenue ayant subi une double mastectomie à la suite d'un cancer du sein n'est pas transsexuelle (assignée homme à la naissance et souhaitant devenir femme), mais elle mobilise la logique de la dysphorie du genre dans le bureau du psychiatre pour que l'État assume les frais associés à sa reconstruction mammaire. En ce qui concerne une détenue lesbienne de l'unité 9, elle émerge d'un coma à la saison 3 et se considère alors un homme. Plutôt que d'être cautionné ou encouragé, le ressenti trans ${ }^{\star}$ du personnage est présenté comme un désir latent et atypique. Il lui vaut un suivi psychologique au cours duquel on lui rappelle qu'elle est une femme. L'identité trans ${ }^{\star} \mathrm{du}$ personnage est éphémère.

Malgré ces lacunes, Unité 9 est d'autant plus pertinente qu'elle fait le lien entre la fiction et la réalité. En novembre 2013, une partie de l'équipe a visité la Maison Tanguay, une prison pour femmes. Plus encore, deux productions ont été diffusées parallèlement à la série: un webdocumentaire comprenant les témoignages de treize femmes détenues et ex-détenues et un documentaire portant sur l'évolution du métier d'IPL (Radio-Canada, 2016). Une page Facebook dédiée à Unité 9 agit comme forum et plateforme de diffusion. Son administratrice, l'auteure Danielle Trottier, invite régulièrement les téléspectateurs à commenter les événements de la série. Ainsi, par sa mouvance du dedans vers le dehors, Unité 9 touche plusieurs consciences. Nous osons espérer que les angles morts de l'incarcération au féminin continueront d'être mis en exergue et que d'autres recherches suivront afin de saisir le potentiel sociologique, critique et transformateur de cette télésérie.

24. La transsexualité de l'infirmière de l'établissement est présentée sous la forme d'un secret. Elle nous est dévoilée progressivement à travers ses activités postopératoires. 


\section{Références}

Baer, L. D. (2005). Visual imprints on the prison landscape: a study on the decorations in prison cells. Tijdschrift voor economische en sociale geografie, 96, 209-217.

Brennan, S. (2014). Canada's Mother-Child Program: Examining Its Emergence, Usage and Current State. Canadian Graduate Journal of Sociology and Criminology, 3(1), 11-33.

Bertrand, M.-A. (1979). La femme et le crime. Montréal, Québec: Les Éditions de l'Aurore.

Bosworth, M. (1999). Engendering Resistance: Agency and Power in Women's Prisons. New York, NY: Ashgate.

Bureau de l'enquêteur correctionnel. (2008). Une mort évitable. Ottawa, Ontario: Bureau de l'enquêteur correctionnel. Repéré à http://www.oci-bec.gc.ca/ cnt/rpt/oth-aut/oth-aut20080620-fra.aspx

Bureau de l'enquêteur correctionnel. (2013-2014). Rapport annuel de l'enquêteur correctionnel 2013-2014. Ottawa, Ontario: Bureau de l'enquêteur correctionnel. Repéré à http://www.oci-bec.gc.ca/cnt/rpt/annrpt/annrpt20132014-fra. aspx

Bureau de l'enquêteur correctionnel. (2014-2015). Rapport annuel de l'enquêteur correctionnel 2014-2015. Ottawa, Ontario: Bureau de l'enquêteur correctionnel. Repéré à http://www.oci-bec.gc.ca/cnt/rpt/pdf/annrpt/annrpt20142015fra.pdf

Butler, J. (1988). Performative Acts and Gender Constitution: An Essay in Phenomenology and Feminist Theory. Theater Journal, 40(4), 519-531.

Butler, J. (2006a). Défaire le genre. Paris, France: Éditions Amsterdam.

Butler, J. (2006b). Trouble dans le genre le féminisme et la subversion de l'identité (traduit de l'anglais par Cynthia Krauss). Paris, France: La Découverte.

Chamberland, C.-A. (2014). Le processus disciplinaire des prisons du Québec: une histoire de logiques. Ottawa, Ontario: Presses de l'Université d'Ottawa.

Chartrand, V. (2015). Landscapes of Violence Women and Canadian Prisons. Revue Champ pénal, 12. Répéré à http://champpenal.revues.org/9158.

Chauvenet, A. (2000). Les surveillants entre droit et sécurité: une contradiction de plus en plus aiguë. Dans C. Veil et D. Lhuiler (dir.), La prison en changement (p. 127-157). Paris, France: Ères.

Clancy, N. (2015, 11 mars). Prison food after cutbacks called disgusting and inadequate by B.C.inmates. CBC News. Repéré à http://www.cbc.ca/news/ Canada/british-columbia/prison-food-after-cutbacks-called-disgustingand-inadequate-by-b-c-inmates-1.2989657.

Clemmer, D. (1958). The prison community. New York, NY: Rinehart.

Correctional Service Canada. (2014). Communiqué to inmates for Pacific Region October $1^{\text {st }} 2014$ Food Service Modernization. CBC News. Repéré à https://www.documentcloud.org/documents/1684635-correctionalservices-canada-memo-on-food.html. 
Corriveau, P. (2007). Discours religieux et médical au cœur du processus de légitimation du droit pénal La gestion des mœurs homoérotiques au Québec (1892-1969). Revue Champ pénal, 4. Repéré à https://champpenal.revues. org/2282\#ftn1.

De Graaf, K. et Kilty, J. M. (2016). You are what you eat: Exploration the relationship between women, food and incarceration. Punishment $\mathfrak{W}$ Society, 18(1), 27-46.

Enos, S. (2001). Mothering from the inside: parenting in a women's prison. Albany, NY: State University of New York Press.

Ferraro, R. (2014, 31 mars). Laverne Cox to be honored at 25th Annual GLAAD Media Awards in Los Angeles. Glaad. Repéré à www.glaad.org/releases/ laverne-cox-be-honored-25th-annual-glaad-media-awards-los-angeleshttp://www.glaad.org/releases/laverne-cox-be-honored-25th-annualglaad-media-awards-los-angeles

Forsyth, C. J., Evans, R. D. et Foster, D. B. (2002). An Analysis of Inmate Explanations for Lesbian Relationships in Prison. International Journal of Sociology of the Family, 30(1/2), 67-77.

Frigon, S. (2000). Corps, féminité et dangerosité: de la production de «corps dociles» en criminologie. Dans S. Frigon et M. Kérisit (dir.), Du corps des femmes contrôles, surveillances et résistances (p. 127-164). Ottawa, Ontario: Presses de l'Université d'Ottawa.

Frigon, S. (2004). Transformation de la philosophie et de la gestion pénale des femmes justiciables au Canada: trois cas de figure. Canadian Journal of Women and the Law, 16(2), 353-385.

Frigon, S. (2009). Écorchées. Montréal, Québec: Les éditions du Remue Ménage.

Frigon, S. (2012). Le corps féminin incarcéré: site de contrôle et de résistance. Dans S. Frigon (dir.), Corps suspect, corps déviant (p. 229-254). Montréal, Québec: Les éditions du Remue-ménage.

Frigon, S. (2013, 10 avril). Les femmes artichauts d'Unité 9. Le Devoir. Repéré à http://www.ledevoir.com/culture/television/375262/les-femmesartichauts-d-unite- 9

Frigon, S. (2014). When Prison Blossoms into Art: Dance in Prison as an Embodied Critical Creative Performative Criminology. Dans M. H. Jacobsen (dir.), The Poetics of Crime: Understanding and Researching Crime and Deviance Through Creative Sources (p. 237-262). Londres, Royaume-Uni: Ashgate Publishing.

Frigon, S. (2015). La danse en criminologie: une échappée belle hors de la classe. Dans M. Cifali, F. Giust-Desprairies et T. Périlleux (dir.), Processus de création et processus cliniques (p. 113-135). Paris, France: Presses universitaires de France.

Garfinkel, H. (1956). Conditions of Successful Degradation Ceremonies. The American Journal of Sociology, 61(5), 420-424.

Giroux, L. et S. Frigon. (2011). Profil correctionnel 2007-2008: les femmes confiées aux Services correctionnels. Québec: Ministère de la Sécurité publique. Direction générale des services correctionnels.

Goffman, E. (1961). Asylum: essays on the social situation of mental patients and other inmates. New York, NY: Double Day and Co. 
Hamelin, M. (1989). Femmes et prison. Montréal, Québec: Éditions du Méridien, 127-168.

Holt, R. et Zundel, M. (2014). Understanding Management, Trade, and Society Through Fiction: Lessons from The Wire. Academy of Management Review, October. 576-585.

Inspectrice en chef des prisons. (2006). Rapport sur une inspection annoncée de l'Établissement Grand Valley pour femmes. Ottawa, Ontario: Services correctionnels Canada. Repéré à http://www.csc-scc.gc.ca/publications/fsw/ wos25_GrandValley/tableOfContents-fra.shtml

Joël-Lauf, M. (2009). L'intimité des femmes incarcérées. Une expérience de terrain. Ethnologie française, 39(3), 547-556. Repéré à www.cairn.info/revueethnologie-francaise-2009-3-page-547.htm

Joël-Lauf, M. (2013). Coûts et bénéfices de l'homosexualité dans les prisons de femmes. Ethnologie française, 3, 469-476.

Killerman, S. (2012). What does the asterisk in "trans"” stand for? Repéré à http:// itspronouncedmetrosexual.com/2012/05/what-does-the-asterisk-in-transstand-for/

Lavigne, B. (1999). Corps et enfermement: récits de femmes. Ottawa, Ontario: Presses de l'Université d'Ottawa.

Leavy, P. (2009). Method meets art: arts-based research practice. New York, NY: Guildford Press.

LeBlanc, N., Kilty, J. M. et Frigon, S. (2015). Examining the preventable but predictable death of Ashley Smith. International Journal of Prisoner Health, 11(3), 126-140.

Mac Farlane, S. (2015, 27 janvier). Laiterie Chagnon perd un important contrat à la prison de Cowansville. Granby Express. Repéré à http://www. granbyexpress.com/Actualites/2015-01-27/article-4022632/LaiterieChagnon-perd-un-important-contrat-a-la-prison-de-Cowansville/1

Martel, J. (2001). Telling the Story. A Study of Segregation with Women Prisoners. Social Justice, 28(1), 196-215.

Milhaud, O. (2015). L'enfermement ou la tentation spatialiste. De «l'action aveugle, mais sûre» des murs des prisons. Annales de Géographie, 2-3(702703), 140-162.

Ministère de la Sécurité publique. (1999). Lois et règlements concernant les personnes prévenues et contrevenantes. Québec: Ministère de la Sécurité publique. Répéré à http://collections.banq.qc.ca/ark: /52327/bs54047

Moran, D., Pallot, J. et Piacentini, L. (2013). Privacy in penal space: Women's imprisonment in Russia. Geoforum, 47(9), 138-146.

Munn, M. et Bruckert, C. (2013). On the outside: From Lengthy Imprisonment to Lasting Freedom. Victoria, Colombie-Britannique: UBC Press Vancouver.

Numéris. (2015). Palmarès des émissions au Québec du 5 au 11 octobre 2015. Repéré à http://assets.numeris.ca/Downloads/October \% 205\% 20\% 20October\% 2011, \% 202015\% 20(Quebec).pdf

Numéris. (2015). Palmarès des émissions au Québec du 10 au 16 septembre 2012. Repéré à http://assets.numeris.ca/Downloads/10\% 20septembre \% 20au \% 2016\% 20septembre \% 202012\% 20(Semaine \% 203)-Quebec.pdf 
Numéris. (2015). Palmarès des émissions au Québec du 31 mars au 6 avril 2014. Repéré à http://assets.numeris.ca/Downloads/31\% 20mars \% 20au \% $206 \%$ 20avril\% 202014\% 20(Semaine\% 2032)-Quebec.pdf.http://assets. numeris.ca/Downloads/31 \% 20mars \% 20au \% 206 \% 20avril\% 202014\% 20(Semaine \% 2032)-Quebec.pdf

Penfold-Mounce, R., Beer, D. et Burrows, R. (2011). The Wire as Social Science-fiction? Sociology, 45(1), 152-167.

Prison Justice.ca. (2016). Transgender Prisoners in Canada. Repéré à http://www. prisonjustice.ca/starkravenarticles/trans_in_prison_0706.html

Public Safety Canada. (2008). Minister Day tightens rules for Mother-Child Program to ensure child protection. Ottawa, Ontario: Public Safety Canada. Repéré à http://www.publicsafety.gc.ca/media/nr/2008/nr2008062 7-1-eng.aspx.

Radio-Canada. (2016). Unité 9 le documentaire. Radio-Canada. Repéré à: http:// unite9.radio-canada.ca/webdoc

Rostaing, C. (1997). La relation carcérale: Identités et rapports sociaux dans les prisons de femmes. Paris, France: Presses universitaires de France.

Service correctionnel Canada. (2007). Programme mère-enfant en établissement. Ottawa, Ontario: Service correctionnel Canada. Repéré à http://www. csc-scc.gc.ca/politiques-et-lois/768-cd-fra.shtml\#ap

Service correctionnel Canada. (2013). Mission du Service correctionnel Canada. Ottawa, Ontario: Service correctionnel Canada. Repéré à http://www. csc-scc.gc.ca/politiques-et-lois/001-cd-fra.shtml.

Service correctionnel Canada. (2015). La création de choix: rapport du groupe d'étude sur les femmes purgeant une peine fédérale, Ottawa, Ontario: Service correctionnel Canada. Repéré à http://www.csc-scc.gc.ca/femmes/choice11e-fra.shtml

Shantz, L. et Frigon, S. (2010). Home free? The (After)Effects of Imprisonment on Women's Bodies, Physical and Mental Health and Identity. Aporia: The Nursing Journal, 2(1), 6-17.

Smoyer, A. B. (2016). Making Fatty Girl Cakes: Food and Resistance in a Women's Prison. The Prison Journal, 96(2), 191-209.

Société Elizabeth Fry du Québec. (2005). Mon guide personnel sur L'isolement préventif et disciplinaire. Repéré à http://www.elizabethfry.qc.ca/wpcontent/uploads/2014/12/Guide_isolement_2005_FR.pdf.

Suto, I. et Arnaut, G. L. Y. (2010). Suicide in Prison: A Qualitative Study. The Prison Journal, 90(3), 288-312.

Sykes, G. (1958/2007). The society of captives: a study of maximum security prison. Princeton, NJ: Princeton University Press.

Therrien, R. (2013, 7 novembre). Les femmes d'Unité 9 à la prison de Tanguay. Le Soleil. Repéré à http://www.lapresse.ca/le-soleil/arts/television-etradio/201311/06/01-4708006-les-femmes-dunite-9-a-la-prison-de-tanguay. php

Walsh, A. (2014). (En)gendering Habitus: Women, Prison, Resistance. Contemporary Theatre Review, 24(1), 40-52.

Wesley, M. (2012). Marginalisées: L'expérience des femmes autochtones au sein des services correctionnels fédéraux. Ottawa, Ontario: Sécurité publique 
Canada. Repéré à www.securitepublique.gc.ca/cnt/rsrcs/pblctns/mrgnlzd/ index-fra.aspx\#s12

Winckler, M. (2009, 2 avril). Ailleurs qu'en France... Les téléfictions sont plus libres, plus engagées, plus subversives que les émissions d'actualité. Repéré à http://www. martinwinckler.com/article.php3?id_article $=945$

\section{The female inmates of Unité 9 : comparing fictional space and reality}

ABSTRACT - In this article Unité 9, a French Canadian television show about women in prison, is analyzed as a way to explore some aspects of women's experience in federal prisons in Canada. After giving a brief history of female incarceration and describing some general characteristics of this prison population, we provide a synopsis of Unité 9 episodes and discuss the relevance, benefits, and limitations of using a television program to help understand actual situations. We then discuss our theoretical understanding of prisons, their dynamics, and the resistance engaged in by women prisoners (Smoyer, 2016; Lavigne, 1999; Frigon, 2000, 2012; Bosworth, 1999; DeGraff et Kilty, 2016 et Joël-Lauf, 2009). Last, we explore some parallels between fiction and reality by looking at four prison sectors: admissions, isolation units, residential areas, and mother-child spaces, revealing different forms of submission, alienation, and resistance. This article sheds light on the inter-play between prison space and female prisoners - both those sentenced federally and those on Unite 9.

KEYWORDS - Space, Unité 9, resistance, sociological leverage, incarcerated women.

\section{Las mujeres detenidas de Unidad 9 : entre espacio ficcional y realidad}

RESUMEN - En el presente artículo, presentamos Unidad 9, una serie de TV quebequense que trata sobre el universo carcelario en femenino, con el fin de trazar paralelismos con la encarcelación de mujeres en la jurisdicción federal.

En premier lugar, exponemos una breve historia de la reclusión femenina y subrayamos algunas particularidades de dicha población penitenciaria. Seguidamente, presentamos la sinopsis de la serie y esbozamos un análisis que subraya la relevancia, los beneficios así como las lagunas de los análisis televisuales. En segundo lugar, teorizamos sobre el espacio carcelario, sobre las dinámicas que alli se instalan y sobre la resistencia emprendida por las mujeres (Butler, 1988, 2006ab; Smoyer, 2016; Lavigne, 1999; Frigon, 2000, 2012; Bosworth, 1999; DeGraff et Kilty, 2016 et Joël-Lauf, 2009). En tercer lugar, establecemos paralelismos entre ficción y realidad explorando cuatro espacios: el sector de las admisiones, el sector del aislamiento, la unidades residenciales y las unidades madre-hijo. Revelamos varios matices de resistencia, de alineación o de sumisión. Así pues, este artículo clarifica la relación entre el espacio penitenciario y las mujeres encarceladas: no solamente aquéllas del distrito federal, sino también aquéllas de la Unidad 9.

PALABRAS CLAVE - Espacio, Unidad 9, impulso sociológico, resistencia, mujeres encarceladas. 